\title{
A review of stereotactic body radiation therapy (SBRT) from the molecular radiobiology perspective
}

\author{
Kathryn L. Brinkman • Bin S. Teh • Bo Xu
}

Received: 10 February 2012 /Accepted: 13 April 2012 / Published online: 4 May 2012

(C) Springer-Verlag 2012

\begin{abstract}
Introduction Stereotactic body radiation therapy (SBRT) is a relatively new therapy being used to treat patients with many types of extracranial tumors and some metastases.

Discussion Clinically, SBRT is well documented and research continues to grow and demonstrate the promising effects of SBRT on patients. Much advancement has been made technically as well, including better techniques for immobilizing patients and tumors. In this paper, emphasis is placed on reviewing the advancements and discoveries of SBRT which are related to molecular radiation biology. These studies include molecular comparisons of high- and low-dose radiation tactics, and molecular methods for improving the outcome of SBRT, including advancements in tumor cell tracking, strategies for increasing the speed of SBRT, and mechanisms behind normal tissue responses after high-dose radiation.

Conclusion Overall, there is still a relatively large amount of basic molecular unknowns involved in SBRT, and continued research is greatly anticipated.
\end{abstract}

Keywords Stereotactic body radiation therapy $\cdot$ Molecular radiobiology $\cdot$ Extracranial tumors

\section{Introduction}

Stereotactic body radiation therapy (SBRT) is a relatively new method established for delivering highly precise and intense doses of radiation to an extracranial tumor site.

K. L. Brinkman • B. S. Teh • B. Xu ( $\bowtie)$

Department of Radiation Oncology,

The Methodist Hospital Research Institute,

6670 Bertner Avenue,

Houston, TX 77030, USA

e-mail: bxu@tmhs.org
SBRT was developed after the establishment of the intracranial version of SBRT, stereotactic radiosurgery (SRS) or fractionated SRS. SRS also delivers high doses of radiation to a targeted volume and was likely introduced first due to the relative ease of immobilizing both patients and tumors. Indeed, while SRS has long been established, SBRT implementation was slower due to the complexity of immobilizing patients or engineering other practices to account for tumor motion during the procedure. After these immobilization strategies were developed, SBRT joined SRS in treating primary tumors by utilizing intense radiation doses, single or fractionated, precisely targeted to a cancerous lesion. This strategy increases tumor cell death, while sparing surrounding healthy tissues.

SBRT has been developed mainly through technical and clinical approaches, leaving a large amount of molecular and cellular information unexplored. Although SBRT continues to perform well in the clinic, understanding the molecular differences between the intense, targeted radiation of SBRT and other forms of therapeutic radiation is essential for refining and enhancing SBRT and extracting the best outcomes possible for patients undergoing SBRT. Other aspects of SBRT could also be enhanced by approaching them from a molecular radiobiology standpoint; such as normal tissue responses to SBRT-like radiation and live tracking of target tumor cells while performing SBRT. All of these topics will be reviewed in this article along with any other pertinent recent developments from the field of molecular radiation biology.

\section{Does the linear quadratic model fit SBRT?}

One primary concern for using SBRT is estimating how much radiation is needed to kill tumor cells located inside 
of a patient's body. The linear quadratic (LQ) model was developed to predict cell survival curves at different doses by incorporating two components of radiation cell killing, one being proportional to the dose, and the other being proportional to the square of the dose.

$S=e^{-\alpha D-\beta D^{2}}$

Notably, $\alpha$ represents lethal lesions, while $\beta$ represents sublethal lesions to DNA within a cell. Although the LQ model accurately predicts cell killing for lower doses of radiation, it has been strongly argued as inappropriate for the high-dose range of radiation used in SBRT due to overestimating cell killing as demonstrated by Timmerman et al. and others [1-3]. This overestimation is related to the predicted amount of sublethal damage. Additionally, the biologically effective dose (BED), which has been used to compare potency and the effect of different dose fractionations of radiation, is derived from the LQ model and fails to account for aspects of tumor survival such of reoxygenation. Nonetheless, many clinicians use these models to predict and translate doses of radiation for their patients due to their simplicity and ease of use [4-7]. Furthermore, other researchers have published reports arguing that the LQ model is appropriate for use in SBRT [8, 9]; however, these reports do not definitively show that the LQ model is the best fit for the high doses of radiation used in their study, and are therefore inconclusive.

Modifications to the LQ model have been encouraged as a solution for the errors produced by using the LQ and BED models. Park et al. developed a new model, deemed the universal survival curve (USC), by integrating two classical radiobiological models [2]. The LQ and the multivariate models combine a straight line and parabola survival curve to produce a more linear relationship at high doses while keeping the sloping curve of the LQ model at low doses. However, the USC model was recently tested (along with others) in vitro by McKenna and Ahmad, and was found to be the most difficult of eight formulae to use when specialized software was unavailable for processing piecewise smooth curves [10]. Nonetheless, the USC curve did provide reliable, accurate predictions of both high- and lowdose cell killing when the required processing technology was available. Wang et al. have also proposed a new model, described as a generalized LQ (gLQ) model, for predicting radiation therapy outcomes [11]. By modifying the predicted amount of sublethal damage induced in the DNA of tumor cells, they were able to make an improved assessment of cell killing compared to the LQ model. Although Wang et al.'s model has yet to be verified by other publications, Huang et al. published another generalized LQ model which is similar in that they modified the sublethal damage component [12]. By altering the sublethal component to include a repair pattern related to reciprocal time, Huang et al. found that their gLQ model also provided better predictions of high-dose cell killing than the LQ model. Using these models to estimate high-dose cell killing may be beneficial for clinical therapy. However, using caution is strongly suggested as the true survival of tumor cells within a patient's body depends on many factors which cannot be accounted for by a mathematical model, such as gene expression differences, tumor blood vasculature and stroma responses, reoxygenation, and immune function.

\section{Molecular comparison of SBRT-like radiation and other types of radiation}

Due to the many forms of radiation used in radiation oncology, determining whether or not there are differences between the effects they have on cells at the molecular level seems critical. The major difference between SBRT and other non-similar forms of radiation is that SBRT uses high dose per fraction radiation, whereas conventional therapy uses low dose per fraction radiation. Additionally, comparing differences between low-dose-rate and high-dose-rate strategies could further optimize SBRT treatment. Additionally, certain cancers with low $\alpha / \beta$ ratios can benefit from decreased radiation times as described by Arcangeli et al. [13].

It is not unexpected that dose-dependent DNA damage responses are induced by ionizing radiation (IR). For example, the maintenance of late $\mathrm{G} 2$ accumulation (the late $\mathrm{G} 2$ cell cycle checkpoint), is dependent on the IR doses [14]. Similarly, different signal transduction pathways in response to IR are likely to be activated dependent on the radiation dose delivered. These differences involve transcriptional, translational, and posttranslational modification regulation of genes and their products, and they are likely to have an impact on combination therapy when molecular targeted radiosensitizers are used in combination with radiation.

In a recent article, Fuks and Kolesnick proposed some finely illustrated molecular nuances between high doses of radiation ( $>8-10 \mathrm{~Gy} /$ fraction) and low doses of fractionated radiation (1.8-3 Gy/fraction) on tumor cell vasculature [15]. In fractionated radiotherapy, endothelial tumor cells undergo radiation induced hypoxia, generate "waves" of reactive oxygen species (ROS) in response, and stimulate translation of hypoxia-inducible factor-1 (HIF-1). In turn, HIF-1 signals angiogenesis stimulating molecules leading to radioresistance of tumor vasculature. Conversely, in single, high-dose radiation, lesions induce the translocation of acid sphingomyelinase (ASMase) which subsequently completes production of ceramide, leading to apoptic signaling and eventual cell death. Moeller et al. published direct evidence of this pathway, first testing the role of HIF-1 in modification of tumor vasculature 
sensitivity to radiation [16]. Moeller et al. found that HIF-1 transcripts were stored in stress granules until reoxygenation by ROS helped trigger release of the HIF-1 transcripts from the stress granules. Once released, the HIF-1 transcripts were translated and the HIF-1 proteins were able to signal proangiogenic factors, such as vascular endothelial growth factor (VEGF) and basic fibroblast growth factor (bFGF), leading to vascular protection and radioresistance. It is interesting to note that these experiments were performed in vivo with radiation doses ranging from 0 to 15 Gy in 5 Gy fractions. As these results strongly suggested HIF-1 to be an ideal target for clinical therapies combined with radiation, Moeller et al. further explored the role of HIF-1 by determining the effect of HIF-1 modulation on overall tumor radiation response [17]. They found that although HIF-1 leads to radioresistance in tumor vasculature, HIF-1 also plays radiosensitizing roles within the tumor cell. Eventually, the authors concluded that although the schedule of HIF-1 inhibition ultimately depends on the specific tumor being treated, concurrent therapy or therapy given between fractions of radiation may provide the best schedule for inhibiting HIF-1. Combined together, these two studies by Moeller et al. provided a good molecular mechanism of how tumor cells respond to fractionated radiotherapy in vivo. More recently, Truman et al. worked on delineating the mechanisms of angiogenesis in high, singledose radiation in vivo [18]. They confirmed ceramidemediated apoptosis as the mechanism of cell death after high-dose radiation, and also found that bFGF and VEGF were capable of inhibiting ASMase activity, thereby causing tumor cell radioresistance. Further, the authors proposed that using anti-angiogenic drugs was more effective immediately before radiation treatment, indicating slightly different drug schedules for fractionated therapy versus ablative single-dose radiotherapy.

One topic which is important for understanding cells' reactions to radiation is that of measuring the amount of damage induced per cell and also visualizing how cells react to this damage. Traditionally, researchers have used staining of DNA damage response (DDR) proteins, such as gamma $\mathrm{H} 2 \mathrm{AX}, 53 \mathrm{BP} 1$ etc. to identify locations of damaged DNA $[19,20]$. DDR proteins congregate around damaged DNA and form what is termed radiation induced foci (RIF), and these RIF can be used as a means to measure the amount of damage that has been induced in a cell. However, in a recent paper, Neurmaier et al. found that determining the amount of damaged DNA in a cell is not best deduced from measuring foci of still images alone [21]. After comparing damaged DNA from images captured using both live cell imaging and still images at many time points, they developed a mathematical model which was capable of calculating the total number of RIF formed in a cell after radiation. The equation provides an accurate number for the total amount of RIF and can be applied using RIF counts from still images. In their own study, Neurmaier et al. used their equation and found that fewer RIF were formed after highdose (1 and 2 Gy) radiation than after low dose (0.1 Gy). High and low doses also differed in how quickly RIF were formed and resolved. High-dose RIF formed relatively faster and resolved slower than low-dose RIF. Additionally, they found that in 2-Gy-treated groups, RIF were brighter. This indicates that on the molecular level, cells respond differently to high doses of radiation by forming larger and more intense clusters of DNA repair proteins, while congregating in fewer locations. The finding that still images are not reliable for assessing the amount of damage induced in a cell has major implications for the way we approach comparing damage induced by different types of radiation. Live cell imaging and/or using the proposed mathematical equation may be best for assessing the amount of damage induced by SBRT in the future.

Recently, a study comparing low-dose-rate (LDR) and high-dose-rate (HDR) radiation was performed by Chen et al. [22]. Two lung cancer cells lines known to have different levels of radiosensitivity to HDR, A549, and NCI-H446 (more sensitive), were exposed to either seeds of ${ }^{125}$ I radiation $(\sim 5 \mathrm{cGy} / \mathrm{h})$ or to gamma rays from a ${ }^{137} \mathrm{Cs}$ irradiator $(0.86 \mathrm{~Gy} / \mathrm{min})$ for total doses of 2 Gy (with or without wortmannin) or $4 \mathrm{~Gy}$ (without wortmannin). Molecularly, they compared differences in expression levels of the proteins $\mathrm{Ku} 80$ and $\mathrm{Ku} 70$ and also the expression level of phosphorylated $\mathrm{Ku} 80 . \mathrm{Ku} 70$ and $\mathrm{Ku} 80$ are two major players in DNA damage repair. These proteins are involved in non-homologous end joining (NHEJ), and together combine to form the $\mathrm{Ku}$ subunit of DNA-dependent protein kinase (DNA-PK). The Ku subunit of DNA-PK functions to bind damaged DNA and to subsequently signal to other NHEJ proteins. Additionally, $\mathrm{Ku}$ is able to activate the other subunit of DNA-PK, the DNA-PK catalytic subunit (DNAPKcs), to promote DNA repair [23]. Therefore, depletion of either $\mathrm{Ku} 70$ or $\mathrm{Ku} 80$ could be expected to promote radiosensitivity, while overexpression could be expected to result in radioresistance. Naturally, this theory has been tested and confirmed by multiple studies [23-26]. Chen et al. used these proteins in their study to determine whether or not $\mathrm{Ku} 70$ or Ku80 expression levels might be responsible for the radiosensitization/resistance seen in their experiments. Additionally, since Ku80 phosphorylation has been demonstrated to be an indicator of the binding activity of $\mathrm{Ku}$ to DNA, Ku80 phosphorylation levels were measured. The authors found that less Ku70 was expressed in NCI-H446 than in A549 cells; however, neither LDR nor HDR had any effect on the expression levels of the Ku proteins in either of the cell lines. For A549 cells, they found that Ku80 phosphorylation increased during all doses of both HDR and LDR. However, for NCI-H446 cells, Ku80 phosphorylation levels increased for all DRs and doses except for 4 Gy LDR 
radiation. The decrease in Ku80 phosphorylation after 4 Gy of LDR radiation indicates malfunction of the DNA repair machinery, and indeed cellular radiosensitization was observed. This means that LDR was more effective at killing lung tumor cells than HDR when using 4 Gy radiation. This interesting observation helps shed some light on a potential molecular mechanism on how cancer cells respond uniquely for different dose rates of radiation and indicates that different dose-rate strategies may be required for SBRT planning. Similar studies using higher doses of radiation on more cancer cell types would be helpful for improving SBRT.

\section{Strategies for enhancing the clinical outcome of SBRT}

\section{Decreasing time spent during SBRT—FFF-VMAT}

The time spent treating patients with radiation during SBRT is relatively long compared with other treatment modalities due to the increased dose, complex procedure, and technical issues, such as patient positioning and tumor movement. This increased "beam-on" time raises clinic-based concerns such as decreased patient comfort and increased chances of delivering off target radiation [27]. At the same time, molecular-based concerns are raised such as increasing the likelihood for cells to repair damage before completion of the full dose, thus causing radioresistance and a decrease in effectiveness of the therapy. Therefore, a strategy which has been proposed and emphasized by many is that of decreasing the length of time cells are exposed to radiation during SBRT [28]. A promising new development in this area is flattening filter-free volumetric modulated arc therapy (FFFVMAT) irradiators, such as the TrueBeam STx from Varian Medical Systems. The difference between FFF-VMAT linear accelerators (LINACs) and other LINACs is easily distinguishable from the name-FFF-VMAT LINACs have had the flattening filter removed, or are made without a flattening filter. By removing the flattening filter, these LINACs are able to produce radiation at extremely HDRs and also deliver more precise radiation [29]. Indeed, Zhang et al. compared the relative speed of FFF-VMAT to noncoplanar 3D and non-coplanar and coplanar VMAT plans in their recent study and confirmed the higher speed of FFFVMAT [30]. Additionally, they compared aspects of plans such as conformity to target, normal tissue dose fall-off, and dose to normal tissue ratio using statistical analyses [30]. In all cases excluding number of monitor units required, Zhang et al. found that the FFF-VMAT plan was statistically superior compared with 3D plans. Since FFF-VMAT and other similar LINACs should greatly benefit SBRT, molecular and cellular studies using and comparing FFF-VMAT with other types of radiotherapy are greatly anticipated.
Live tumor cell imaging

From a molecular standpoint, many advances have been made in in vitro live cell imaging using fluorescent labels and have been reviewed extensively elsewhere [31-36]. Almost any protein can be tagged and imaged fluorescently in a variety of living cells by using integrated microscope and incubator systems, such as the IncuCyte system by Bucher Biotec. However, this technique involves transfecting cancer cells with non-native forms of a protein-tag combination and is not feasible to apply clinically. Imageguided radiotherapy and intensity-modulated therapy have been incorporated with SBRT successfully to aid in tracking tumor location. However, further developments could help improve these imaging processes, such as labeling tumor cells fluorescently. Since the benefit of being able to image tumor cells while administering radiation therapy is clear, researchers have been exploring new strategies for imaging normal and tumor cells using translation-orientated in vivo studies.

In several recent studies, nanoparticles have been used to image cells in vivo. In a recent article published by Yang et al., the authors used folate and FITC-conjugated nanoparticles to image and target folate receptor positive tumor cells [37]. Quantum dots (QDs) have also emerged as an intriguing method for imaging tumors in vivo. Quantum dots are semiconductor fluorophore nanomolecules, making them ideal for imaging. Iyer et al. demonstrated this concept in a recent study using protein-coated QDs (pcQDs) to create ideal fluorescent probes which easily conjugate to substrates [38]. Although in vivo investigations using Iyer et al.'s pcQDs are necessary before considering clinical applications, the enhanced stability of Yang et al.'s pcQDs could make them a very effective imaging and targeting tool for use with SBRT.

A recent article incorporating live cell imaging and radiotherapy not only demonstrated the use of singlewalled carbon nanotubes in imaging tumor cells, but also demonstrated using the nanotubes to target tumor cells using photothermal therapy in mice [39]. The idea of using hyperthermia in combination with radiation for treating radioresistant tumors has been explored with many positive results [40-43]. Most recently, Atkinson et al. used gold nanoshells to eliminate cancer stem cells from breast cancer using a combination of hyperthermia and radiation treatment in mice [44]. Nonetheless, the practice of using hyperthermia in conjunction with radiotherapy must be approached with caution due to the potential complication of acute toxicities. Further research to enable using the imaging and photothermal properties of these carbon nanotubes in the clinical setting may be beneficial for use with SBRT, especially for targeting radioresistant cancer stem cells. 
Normal tissue response

While SBRT is superior in greatly reducing the volume of surrounding tissues that will receive radiation, it is inevitable that high doses of radiation will still cause damage to normal tissues. Pulmonary is one prime example of easily damaged tissue, as complete tumor movement control is challenging. One difficulty to surmount in studying normal tissue response is that of an appropriate translational model. Recently, several laboratories have developed promising systems to address this problem in the form of small animal radiation research platforms (SARRPs) [45-48], the development of which stemmed from previously developed small animal micro-radiotherapy systems lacking image guidance $[49,50]$. Zhou et al. have demonstrated the use of their SARRP for lung cancer radiotherapy in mice. The SARRP of Matinfar et al. is a complex machine capable of delivering precise doses (beam diameter as small as $0.5 \mathrm{~mm}$ ) of radiation to mice, thus enabling extensive studies for SBRTinduced normal tissue damage. These newly developed animal models should prove to be very beneficial for future translational studies on protecting healthy tissues in animals receiving ablative radiotherapy.

Although identifying molecular indicators of acute normal tissue response in response to traditional radiotherapy has been extensively studied, information in the SBRT setting is limited. Several DNA damage induced markers, such as gamma $\mathrm{H} 2 \mathrm{AX}$ or 53BP1, have been suggested to be potential indicators of normal tissue toxicity, however, a recent study challenged this concept [51]. Werbrouck et al. attempted to determine the propensity of healthy $\mathrm{T}$ lymphocytes to undergo acute tissue complications by scoring gamma H2AX foci from ATM-deficient lymphoblastoid cell lines and from T-lymphocyte cells extracted from 31 head and neck cancer patients treated by intensity-modulated radiation therapy. The cells collected were irradiated with high-dose-rate $0.5-\mathrm{Gy}$ radiation. They found that gamma $\mathrm{H} 2 \mathrm{AX}$ foci formation in non-cancerous $\mathrm{T}$ lymphocytes from cancer patients was unable to predict whether or not patients would develop acute tissue complications. Therefore, more studies investigating potential molecular markers of risk for tissue toxicity would be especially helpful for SBRT.

\section{Conclusions}

SBRT is an exciting treatment modality that has been clinically proven to be beneficial to many cancer patients. The high-intensity, targeted radiation from SBRT is not only able to spare more healthy tissue, but has been demonstrated to locally control tumors better than when using conventional radiotherapy [28]. Although there are limited studies directly associated with SBRT, several molecular and cellular studies that might have an influence on SBRT were reviewed here. However, it is clear that more molecular and cellular studies are needed to fully understand the mechanisms behind tumor damage and healthy tissue response in high-dose treatment modalities such as SBRT. Indeed, the clinical aptitude of SBRT should encourage molecular radiobiology researchers to investigate the molecular mechanisms behind this promising treatment modality further. SBRT has proven to be a powerful cancer treatment tool, and is sure to only improve with advancements in our understanding of the molecular mechanisms behind this therapy.

Conflict of interest None.

\section{References}

1. Timmerman RD et al (2007) Stereotactic body radiation therapy in multiple organ sites. J Clin Oncol: Off J Am Soc Clin Oncol 25 (8):947-952

2. Park $C$ et al (2008) Universal survival curve and single fraction equivalent dose: useful tools in understanding potency of ablative radiotherapy. Int J Radiat Oncol Biol Phys 70(3):847-852

3. Shibamoto Y et al. (2012) Radiobiological evaluation of the radiation dose as used in high-precision radiotherapy: effect of prolonged delivery time and applicability of the linear-quadratic model. J Radiat Res 53:1-9

4. Wulf J et al (2005) Dose-response in stereotactic irradiation of lung tumors. Radiother Oncol: J Eur Soc Ther Radiol Oncol 77 (1):83-87

5. Milano MT et al (2008) Descriptive analysis of oligometastatic lesions treated with curative-intent stereotactic body radiotherapy. Int J Radiat Oncol Biol Phys 72(5):1516-1522

6. Onishi H et al (2011) Stereotactic body radiotherapy (SBRT) for operable stage I non-small-cell lung cancer: can SBRT be comparable to surgery? Int J Radiat Oncol Biol Phys 81(5): $1352-1358$

7. Takeda A et al (2009) Stereotactic body radiotherapy for primary lung cancer at a dose of 50 Gy total in five fractions to the periphery of the planning target volume calculated using a superposition algorithm. Int J Radiat Oncol Biol Phys 73(2):442-448

8. Fowler JF et al (2004) A challenge to traditional radiation oncology. Int J Radiat Oncol Biol Phys 60(4):1241-1256

9. Brenner DJ (2008) The linear-quadratic model is an appropriate methodology for determining isoeffective doses at large doses per fraction. Semin Radiat Oncol 18(4):234-239

10. McKenna FW, Ahmad S (2009) Fitting techniques of cell survival curves in high-dose region for use in stereotactic body radiation therapy. Phys Med Biol 54(6):1593-1608

11. Wang JZ et al (2010) A generalized linear-quadratic model for radiosurgery, stereotactic body radiation therapy, and high-dose rate brachytherapy. Sci Transl Med 2(39):39ra48

12. Huang $Z$ et al (2012) A generalized linear-quadratic model incorporating reciprocal time pattern of radiation damage repair. Med Phys 39(1):224

13. Arcangeli $\mathrm{G}$ et al (2010) A prospective phase III randomized trial of hypofractionation versus conventional fractionation in patients with high-risk prostate cancer. Int J Radiat Oncol Biol Phys 78 (1):11-18 
14. Xu B et al (2002) Two molecularly distinct $\mathrm{G}(2) / \mathrm{M}$ checkpoints are induced by ionizing irradiation. Mol Cell Biol 22(4):1049 1059

15. Fuks Z, Kolesnick R (2005) Engaging the vascular component of the tumor response. Cancer cell 8(2):89-91

16. Moeller BJ et al (2004) Radiation activates HIF-1 to regulate vascular radiosensitivity in tumors: role of reoxygenation, free radicals, and stress granules. Cancer cell 5(5):429-441

17. Moeller BJ et al (2005) Pleiotropic effects of HIF-1 blockade on tumor radiosensitivity. Cancer cell 8(2):99-110

18. Truman JP, et al (2010) Endothelial membrane remodeling is obligate for anti-angiogenic radiosensitization during tumor radiosurgery. PloS one. 5(9):e12310. doi:10.1371/journal.pone. 0012310

19. FitzGerald JE, Grenon M, Lowndes NF (2009) 53BP1: function and mechanisms of focal recruitment. Biochem Soc Trans 37(Pt 4):897-904

20. Bekker-Jensen S, Mailand N (2010) Assembly and function of DNA double-strand break repair foci in mammalian cells. DNA repair 9(12):1219-1228

21. Neumaier T et al (2012) Evidence for formation of DNA repair centers and dose-response nonlinearity in human cells. Proc Natl Acad Sci U S A 109(2):443-448

22. Chen $\mathrm{H}$ et al. (2011) Comparison of cellular damage response to low-dose-rate (125)I seed irradiation and high-dose-rate gamma irradiation in human lung cancer cells. Brachytherapy 11:149-156

23. Mahaney BL, Meek K, Lees-Miller SP (2009) Repair of ionizing radiation-induced DNA double-strand breaks by non-homologous end-joining. Biochem J 417(3):639-650

24. Yang QS et al (2008) ShRNA-mediated Ku80 gene silencing inhibits cell proliferation and sensitizes to gamma-radiation and mitomycin C-induced apoptosis in esophageal squamous cell carcinoma lines. J Radiat Res 49(4):399-407

25. He F et al (2007) Adenovirus-mediated expression of a dominant negative $\mathrm{Ku} 70$ fragment radiosensitizes human tumor cells under aerobic and hypoxic conditions. Cancer Res 67(2):634-642

26. Ayene IS, Ford LP, Koch CJ (2005) Ku protein targeting by Ku70 small interfering RNA enhances human cancer cell response to topoisomerase II inhibitor and gamma radiation. Mol Cancer Ther 4(4):529-536

27. Roa DE et al (2012) The use of RapidArc volumetric-modulated arc therapy to deliver stereotactic radiosurgery and stereotactic body radiotherapy to intracranial and extracranial targets. Med Dosim: Off J Am Assoc Med Dosim. doi:10.1016/j.meddos.2011.09.005. Accessed 24 February 2012

28. Chang BK, Timmerman RD (2007) Stereotactic body radiation therapy: a comprehensive review. Am J Clin Oncol 30(6):637-644

29. Hrbacek J, Lang S, Klock S (2011) Commissioning of photon beams of a flattening filter-free linear accelerator and the accuracy of beam modeling using an anisotropic analytical algorithm. Int J Radiat Oncol Biol Phys 80(4):1228-1237

30. Zhang GG et al (2011) Volumetric modulated arc planning for lung stereotactic body radiotherapy using conventional and unflattened photon beams: a dosimetric comparison with $3 \mathrm{D}$ technique. Radiat Oncol 6:152

31. Misteli T, Spector DL (1997) Applications of the green fluorescent protein in cell biology and biotechnology. Nat Biotechnol 15 (10):961-964

32. Welsh S, Kay SA (1997) Reporter gene expression for monitoring gene transfer. Curr Opin Biotechnol 8(5):617-622
33. Prasher DC (1995) Using GFP to see the light. Trends Genet: TIG 11(8):320-323

34. Muller-Taubenberger A, Anderson KI (2007) Recent advances using green and red fluorescent protein variants. Appl Microbiol Biotechnol 77(1):1-12

35. Ni Q, Titov DV, Zhang J (2006) Analyzing protein kinase dynamics in living cells with FRET reporters. Methods 40(3):279-286

36. Umezawa Y (2005) Genetically encoded optical probes for imaging cellular signaling pathways. Biosens Bioelectron 20(12):2504 2511

37. Yang $\mathrm{H}$ et al (2011) Investigation of folate-conjugated fluorescent silica nanoparticles for targeting delivery to folate receptor-positive tumors and their internalization mechanism. Intern J Nanomedicine 6:2023-2032

38. Lacoste TD et al (2000) Ultrahigh-resolution multicolor colocalization of single fluorescent probes. Proc Natl Acad Sci U S A 97 (17):9461-9466

39. Robinson JT et al (2010) High performance in vivo near-IR (>1 mum) imaging and photothermal cancer therapy with carbon nanotubes. Nano Res 3(11):779-793

40. Franckena M et al (2009) Radiotherapy and hyperthermia for treatment of primary locally advanced cervix cancer: results in 378 patients. Int J Radiat Oncol Biol Phys 73(1):242-250

41. Gabriele P et al (2009) Radio hyperthermia for re-treatment of superficial tumours. Intern J Hyperth: Off J Eur Soc Hyperthermic Oncol, North Am Hyperth Group 25(3):189-198

42. Jones EL et al (2005) Randomized trial of hyperthermia and radiation for superficial tumors. J Clin Oncol: Off J Am Soc Clin Oncol 23(13):3079-3085

43. Vernon CC et al (1996) Radiotherapy with or without hyperthermia in the treatment of superficial localized breast cancer: results from five randomized controlled trials. International collaborative hyperthermia group. Int J Radiat Oncol Biol Phys 35(4):731744

44. Atkinson RL et al (2010) Thermal enhancement with optically activated gold nanoshells sensitizes breast cancer stem cells to radiation therapy. Sci Transl Med 2(55):55ra79

45. Matinfar $M$ et al (2010) Robotic delivery of complex radiation volumes for small animal research. IEEE International Conference on Robotics and Automation : ICRA : [proceedings] IEEE International Conference on Robotics and Automation, 2010: 20562061.

46. Wong $\mathrm{J}$ et al (2008) High-resolution, small animal radiation research platform with $\mathrm{x}$-ray tomographic guidance capabilities. Int J Radiat Oncol Biol Phys 71(5):1591-1599

47. Zhou H et al (2010) Development of a micro-computed tomography-based image-guided conformal radiotherapy system for small animals. Int J Radiat Oncol Biol Phys 78(1):297-305

48. Graves EE et al (2007) Design and evaluation of a variable aperture collimator for conformal radiotherapy of small animals using a microCT scanner. Med Phys 34(11):4359-4367

49. Stojadinovic $\mathrm{S}$ et al (2007) MicroRT-small animal conformal irradiator. Med Phys 34(12):4706-4716

50. Wang $\mathrm{S}$ et al (2007) A novel high resolution micro-radiotherapy system for small animal irradiation for cancer research. Biofactors 30(4):265-270

51. Werbrouck J et al (2011) Lack of a correlation between gamma$\mathrm{H} 2 \mathrm{AX}$ foci kinetics in lymphocytes and the severity of acute normal tissue reactions during IMRT treatment for head and neck cancer. Int J Radiat Biol 87(1):46-56 